Urologe 2011 · 50:416

DOI 10.1007/s00120-010-2475-5

Online publiziert: 8. April 2011

(c) Springer-Verlag 2011

\author{
U. Otto ${ }^{1} \cdot$ W. Vahlensieck ${ }^{2}$ \\ ${ }^{1}$ Abteilung Urologie u. Onkologie, Klinik Quellental, \\ Kliniken Hartenstein Bad Wildungen, Bad Wildungen \\ ${ }^{2}$ Abteilung Urologie, Onkologie u. Nephrologie, Klinik Wildetal, \\ Kliniken Hartenstein Bad Wildungen, Bad Wildungen
}

\title{
Zur Bedeutung der Rehabilitation urologischer und nephrologischer Erkrankungen
}

erkennung, Diagnose und Therapie der verschiedenen Stadien des Prostatakarzinoms" implementiert werden $[1,2]$.

So stehen dem Rehabilitationsmediziner umfangreiche Daten zur Verfügung, die den Verlauf nach der Prostatastanzbiopsie, insbesondere im Hinblick auf die Frühkontinenz darstellen. Dieses wird in einem Artikel beleuchtet. Im Fokus der Betrachtungen stehen dann Ergebnisse der Rehabilitation nach radikaler Prostatektomie, insbesondere die postoperativen Funktionsstörungen wie die Harninkontinenz, durchaus auch abhängig vom Alter und die erektile Dysfunktion. Auch das umstrittene Thema des Umgangs mit dem Hypogonadismus bei Patienten mit einem Prostatakarzinom ist Inhalt unserer Betrachtungen.

Wir freuen uns, dass wir für die Betrachtungen über die urologische Rehabilitation ausgewiesene Experten gewinnen konnten. Dabei können wir Ihnen natürlich nur einen kleinen Einblick zeigen, von dem, was uns in der stationären Rehabilitation beschäftigt und was sie zu leisten vermag, denn sie ist facettenreich.

Allen Beteiligten, die an diesem Heft mitgewirkt haben, gilt unser Dank für Ihren Einsatz. Die nachfolgenden Artikel mögen dazu beitragen, dass für die Leser die Rehabilitation in einem anderen Licht erscheint.

Wir hoffen, Ihr Interesse geweckt zu haben!

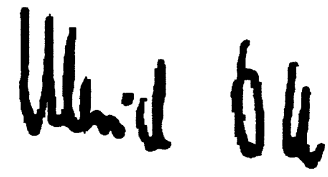

U. Otto

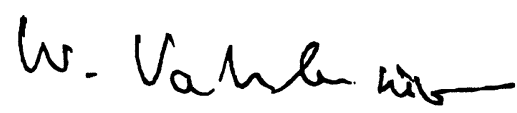

W. Vahlensieck

\section{Korrespondenzadressen Prof. Dr. U. Otto}

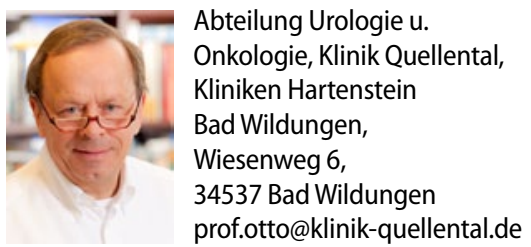

\section{PD Dr. W. Vahlensieck}

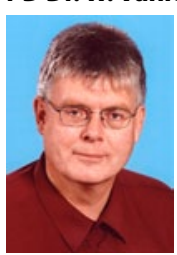

Abteilung Urologie, Onkologie u. Nephrologie, Klinik Wildetal, Kliniken Hartenstein Bad Wildungen, Mühlenstr. 8, 34537 Bad Wildungen winfried.vahlensieck@ t-online.de

\section{Literatur}

1. DGU (2009) Interdisziplinäre Leitlinie der Qualität S3 zur Früherkennung, Diagnose und Therapie der verschiedenen Stadien des Prostatakarzinoms. AWMF-Leitlinien, Register Nr. 043-022

2. Vahlensieck W, Gäck M, Gleißner J et al (2005) Struktur- und Prozessqualität der stationären urologischen Rehabilitation. Urologe A 44:51-56

3. Sozialgesetzbücher V § 40, VI § 15 und 31, IX § 9 und 26 (2010) www.sozialgesetzbuch-sgb.de 